\title{
Gastric stromal tumors: clinical presentation and surgical options
}

\author{
C. Pardo Martínez, J. Mayol Martínez, C. Hernández Pérez and J. Álvarez Fernández-Represa \\ Service of General Surgery I. Hospital Clínico San Carlos. Madrid. Spain
}

\begin{abstract}
Stromal tumors represent $1-3 \%$ of all primary gastric neoplasms. These tumors can occur at any age and display different clinical manifestations, but they rarely reach over $10 \mathrm{~cm}$ in size. Currently they are designed as gastrointestinal stromal tumors (GIST) but their classification is still controversial. Surgery is the treatment of choice, and the extension of surgical resection depends on the tumor size, neoplastic involvement of adjacent organs, and the presence of metastatic disease. In selected cases minimally invasive surgery can provide excellent results.

We present four new patients with GIST who exemplify the different clinical forms of presentations of GISTs and their diverse treatment options.
\end{abstract}

Key words: Leiomyoblastoma. Stromal tumors. GIST. Treatment. Surgery.

Pardo Martínez C, Mayol Martínez J, Hernández Pérez C, Álvarez Fernández-Represa J. Gastric stromal tumors: clinical presentation and surgical options. Rev Esp Enferm Dig 2004; 96: $578-583$

\section{INTRODUCTION}

Gastrointestinal stromal tumors (GISTs) are extremely rare, and represent between 1 and $3 \%$ of all primary gastric neoplasms (1). These tumors may occur at any age but their peak incidence is during the sixth decade of life. The most frequent localization is the mid part of the stomach, followed by the antrum. In almost $20 \%$ of patients the tumor

Recibido: 28-07-03.

Aceptado: 07-10-03.

Correspondencia: Cristina Pardo Martínez. Servicio de Cirugía I. Hospital Clínico San Carlos. Dr. Martín Lagos, s/n. 28040 Madrid. e-mail: crispardomar@hotmail.com appears near the pylorus, although gastric outlet obstruction secondary to neoplastic growth is uncommon (2). GISTs may vary in size between a few milimeters and $20 \mathrm{~cm}$ or more, but only $20 \%$ of them may reach a diameter over 10 $\mathrm{cm}$. Up to $30-50 \%$ of patients with GISTs are asymptomatic $(3,4)$ at the time of diagnosis, particularly those with small tumors. Since the introduction of CT and ultrasound imaging the difficulties to diagnose these tumors by using noninvasive methods have been greatly overcome (5). Both imaging modalities, together with endoscopic examination with multiple biopsies of the suspected lesion (6) for further immunohistochemical studies, are the most valuable tools for the diagnosis of these neoplasms. They must be used not only for the diagnosis and staging of the primary tumor but also for postoperative follow-up.

Curative surgical resection is the treatment of choice for gastric stromal tumors irrespective of whether they are benign or malignant. However, technical options may vary from a minimally invasive approach to massive gastrointestinal exeresis aimed at achieving disease-free margins.

We report four patients whose clinical scenarios represent the multiple diagnostic and therapeutic options for these neoplasms.

\section{CASE REPORTS}

Case 1. A 76-year-old asymptomatic male patient was diagnosed with an extraluminal gastric lump on routine abdominal ultrasound examination. An upper gastrointestinal endoscopy (Fig. 1) showed an extramucosal gastric tumor of $3 \mathrm{~cm}$ in size. He underwent an endoscopy-aided laparoscopic gastric wedge resection. Histopathological examination found a neoplastic mesenchymal proliferation with scarce mitoses (fewer than 1/50 high power field). Immunohistochemically, cells were negative for cytokeratin, S-100, desmin and actin, and positive for vimentin. The patient was free of disease at two years and a half after surgery.

Case 2. A 72-year-old female patient presenting with upper gastrointestinal bleeding was diagnosed with a $4 \mathrm{~cm}$ ul- 




Fig. 1.- A $2 \mathrm{~cm}$-submucosal lesion covered by normal mucosa is observed in the great curvature of the stomach.

Frente a curvatura menor se observa una lesión submucosa de unos 2 $\mathrm{cm}$ de tamaño, cubierta por mucosa gástrica de aspecto normal.

cerated submucosal tumor located in the gastric greater curvature on upper endoscopic examination. She underwent curative open gastric wedge resection with disease-free margins. The histopathological diagnosis was consistent with a gastrointestinal stromal tumor of undetermined malignant potential. After two years of follow-up extragastric tumor recurrence was found on a CT scan, and she underwent "enbloc" resection of the antrum, the great omentum and the spleen. No liver metastases were found. Histopathologically, there were peritoneal, omental, gastric and lymph node metastases from a malignant GIST (over 15 mitoses per high power field). It was positive for CD34 and C-KIT (CD117), and negative for muscle markers (actin and desmin) and for S-100, synaptophysin, and Leu-7. The patient is disease-free at 9 months after reoperation.

Case 3. A 71-year-old patient presented at the emergency department with asthenia, weight loss and intermittent abdominal pain for the last 2 months. On the abdominal CT scan a large abdominal tumor of $25 \times 25 \times 13 \mathrm{~cm}$ in size with necrotic areas was seen. It extended from the epigastrium downwards to the mesogastrium, thus displacing the stomach but without a defined site of origin. There were no liver implants. The patient underwent elective surgical treatment. An "en-bloc" resection of the stomach and transverse colon was carried out due to the presence of a gastric stromal tumor invading the colon. The histopathological study showed a mesenchymal neoplastic proliferation with 31 mitoses per 50 high power field. Neoplastic cells were negative for cytokeratin, actin, desmin, and S-100, and positive for vimentin. There was no lymphatic involvement. The patient was admitted to the emergency department 8 months after surgery because of severe anorexia and a painful abdominal mass on the upper abdomen. An abdominal CT scan (Fig. 2) showed a local tumor recurrence with lymph node, retroperitoneal, mesenteric, liver and pulmonary metastases. The patient died one month later.

Case 4. A 60-year-old female patient with a previous history of high blood pressure presented to the emergency de-

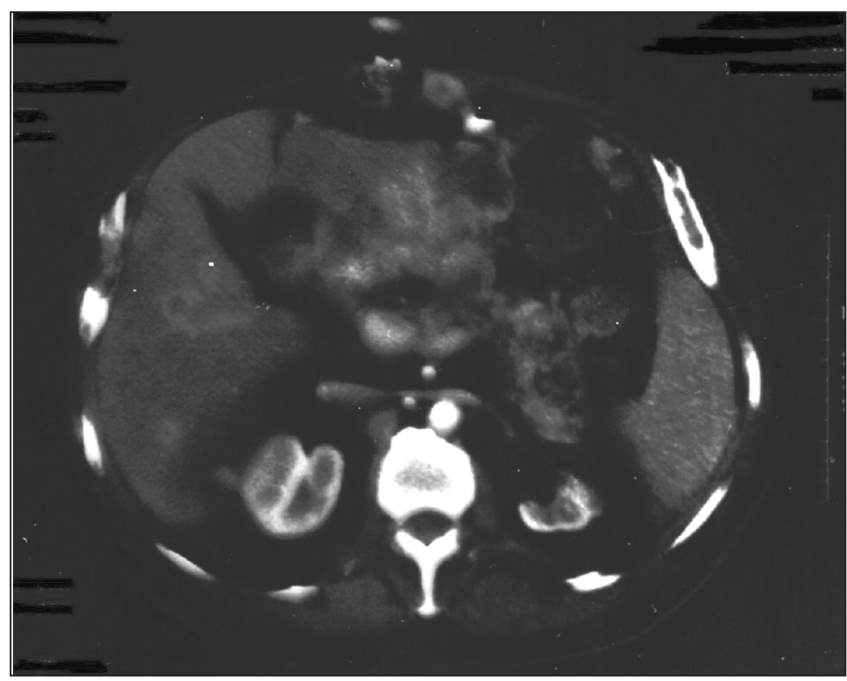

Fig. 2.- Large abdominal mass in close contact with the posterior gastric wall, containing heterogenous areas suggesting bleeding and necrosis.

Masa de grandes dimensiones en íntimo contacto con la pared posterior del estómago, con imágenes en su interior sugerentes de sangrado $y$ necrosis.

partment with acute abdominal pain accompanied by nausea, vomiting, and hypotension. On physical examination she appeared hypotensive (blood presure 60/40). Her hemoglobin concentration was $8.1 \mathrm{~g} / \mathrm{dl}$, and the hematocrit was 24.7\%. An abdominal CT scan (Fig. 3) showed a heterogeneous mass (both solid and liquid) of about $20 \mathrm{~cm}$ in size emerging from the posterior aspect of the stomach. She



Fig. 3.- Extraluminal mass of approximately $25 \mathrm{~cm}$ in diameter, which may be arising from the posterior gastric wall, infiltrating the transverse colon and the distal ileum (1) with extensive necrotic areas (2) and massive hemoperitoneum.

Masa extraluminal de unos $25 \mathrm{~cm}$ de diámetro que parece depender de la pared posterior del estómago y que infiltra el colon transverso y el íleon (1) con extensas áreas de necrosis en su interior (2) y hemoperitoneo masivo. 
underwent emergency surgical treatment for a massive hemoperitoneum and a large necrotic tumor of the stomach invading the distal ileum, the cecum, and both the ascending and transverse colon. Histopathologically, the tumor contained cells organized in bundles with scarce mitoses (fewer that 1/50 high power field) and with a low proliferative index (Ki67). The immunohistochemical study showed that neoplastic cells were negative for actin, desmin, S-100, and keratin, and positive for CD-34 and vimentin. The patient is free of disease at 18 months after surgery.

\section{DISCUSSION}

GISTs represent the larger group of non-epithelial neoplasms of the stomach and the small bowel (7-9). The name GIST was first used by Mazur and Clark (10) in 1983, and this category includes an heterogeneous group of tumors with a wide variety of cellular differentiation. This fact has brought some degree of confusion with regard to its interpretation. In the 1980s several immunohistochemical studies showed that these tumors may display extremely variable cellular differentiation (11-14). They may display smooth muscle (as shown by the expression of actin, myosin and desmin), autonomic nerve, ganglionic or neural differentiation. They may also remain poorly differentiated.

In 1998 Kindblom (15) postulated for the first time the possibility that these tumors may derive from the interstitial cells of Cajal, based on the fact that both cell types are positive for the same marker - CD117 (c-KIT).

Several prognostic systems have been proposed, but the one revised in 1993 by UICC, which comprises tumor size, lymph node involvement, presence of distant metastases and immunopathologic examination, is most widely accepted (16). Prognostic factors that are currently accepted include: tumor location (10), tumor size over $5 \mathrm{~cm}$ (17), immunohistochemical tumor features (nuclear abnormalities, necrosis, vascular invasion, number of mitoses greater than $5 \times 50$ high power field, c-KIT mutation) $(18,19)$, involvement of adjacent organs, and presence of metastases (20). Although several authors have found an association between exon 11 mutations in c-kit gene and the more aggressive behavior of these tumors, recently, Bernett et al (21) were not able to confirm this relationship. Some authors also consider tumor rupture during surgery and incomplete surgical resection as risk factors for poor prognosis (22).

The liver is the most frequent site for metastasis, as seen in case 2, followed by the peritoneum and the lung. Metastatic disease may appear as late as 30 years after resection of the primary tumor. Overall, removal of metastatic disease is recommended because it may increase survival, which is about $50 \%$ at 5 years.

Surgery is the treatment of choice for GISTs. No difference in survival at 5 and 10 years (23) has been observed between wide and more limited surgical resection in patients with tumors under $5 \mathrm{~cm}$ in diameter and no liver metastases at the time of diagnosis. In these patients, as in case 1, a minimally invasive approach may yield excellent results.

GISTs are resistant to both conventional chemotherapy and radiation $(24,25)$. For patients who have undergone non-curative surgery, imatinib mesylate $\left(\right.$ Gleevec $\left.^{\circledR}\right)$ has been recently approved. This drug is in clinical use for the treatment of Philadephia (+) chronic myeloid leukemia. Imatinib mesylate works by blocking the mutated membrane receptor c-KIT, which has intrinsic tyrosine-kinase activity. This mutation is critical for the development of these tumors. By blocking this receptor, the drug blocks tumor proliferation (26) and induces cell apoptosis (27).

Recent studies (28) have shown that a subgroup of GISTs lacks the c-KIT mutation but displays a mutation of the platelet-derived growth factor receptor alpha (PDGFRA), which possesses intrinsic tyrosine-kinase activity. This mutation is identified by immunoprecipitation with polyclonal antibodies against polypeptides with specific sequences of the tyrosine-kinase receptor family. Both mutations, c-KIT and PDGFRA, appear to be alternative and mutually exclusive. Therefore, the absence of a c-KIT mutation does not exclude the diagnosis of GIST and warrants further study of a PDGFRA mutation. If a stromal tumor does not express the c-KIT mutation, Gleevec ${ }^{\circledast}(28)$ may still be considered a therapeutic option. In both preclinical (29) and clinical studies (30) imatinib mesylate has been shown to be effective for patients with unresectable or metastatic disease, thus representing the only available therapeutic modality for these patients.

\section{REFERENCES}

1. Cocco P, Ottomello R, Gemini S, Maxia G, Cdoni S. Il leiomioblastoma gástrico. Pressentazione di un caso. Minerva Chir 1992; 47: 1727-30.

2. Delgado Millán MA, Abad Barahona A, Morales Gutiérrez C, Martín Díaz M, López López A, Sierra García A, et al. Leiomiosarcoma gástrico, a propósito de un caso. Rev Esp Enferm Dig 1991; 80: 213.

3. Palmiere B, Cogni P, Criscuolo M, Di Gregorio C. Leiomioblastoma dello stomaco: Ulteriore contributo casistico. Minerva Chir 1991; 46: 561-6.

4. Sacco D, Paron L, Bardella R, Spidalieri G, Cardino L. Leiomiosarcoma gástrico. Osservazione di tre casi clinici in urgenza. Minerva Chir 1994; 49: 995-9.

5. Poza P, Pombo P, de Juan Rumero P, Gómez-Chao de Santos T, Montero Gómez M. Hipoglucemia asociada a un leiomiosarcoma gástrico. Rev Esp Enferm Dig 1996; 88: 359-60.

6. Zullo A, Botto G, Demaria G. Leiomioblastoma gastrico epitelioide. Descrizione di un caso clinico e considerazioni. Minerva Chir 1994; 49: $207-10$

7. Appelman HD, Helwig EB. Gastric epithelioid leiomyoma and leiomyosarcoma (leiomyoblastoma). Cancer 1976: 709-28.

8. Manconi A. Su un caso de leiomioblastoma dello stomaco. Minerva Med. 1990; 81 (Supl. 7-8): 71-4.

9. Cattaneo U, Andreone D, Enrico S, Serra GC, Toppino M, Corno F, et al. Il rabdomiosarcoma: presentazione di un caso a localizzazione addominale. Minerva Chir 1991; 46: 417-20.

10. Crosby JA, Catton CN, Davis A, et al. Malignant Gastrointestinal Stromal Tumors of the Small Intestine: A review of 50 cases fron a prospective database. Annals of Surgical Oncology 2001; 8: 50-9.

11. Rosay J. Gastrointestubak tract: stromal tumors. En: Ackerman's. Surgical Pathology. $8^{\mathrm{a}}$ ed. Mosby, 1996. p. 645-7.

12. Miettinen M. Gastrointestinal stromal tumors. An immunohistochemical study of cellular differentiation. Am J Clin Pathol 1988 (89): 60110.

13. Franquemont DW. Diferentiation and risk assessment of gastrointestinal stromal tumors. Am J Clin Pathol 1995; 103: 41-7.

14. Suster S. Gastrointestinal stromal tumors. Semin Diagn Pathol 1996; 13: $297-313$. 
15. Kindblom LG, Remotti HE, Aldemborg F: Gastrointestinal pacemaker cell tumor (GIPACT). Gastrointestinal stromal tumors show phenotypic characteristics of the interstitial cells of Cajal. Am J Pathol 1998; 152: 1259-69.

16. Hermanek P, Henson DE, Hutter RVP, Sobin LH. IUCC TNM Suplement 1993: A commentary on Uniform Use. Berlin, 1993: Springer. Verlag.

17. DeMatteo RP, Lewis JJ, Leung D, Mudan SS, Woodruff JM, Brenn MF. Two hundred gastrointestinal stromal tumors: recurrence patterns and prognostic factors for survival. Ann Surg 2000; 231: 51-8.

18. Yan H, Marchettini P, Acherman YI, Gething SA, Brun E, Sugarba PH. Prognostic assessment of gastrointestinal stromal tumor. Am J Clin Oncol 2003; 26: 221-8

19. Singer S, Rubin BP, Lux ML, Chen CJ, Demetri GD, Fletcher CD, et al. Prognostic value of KIT mutation type, mitotic activity, and histologic subtype in gastrointestinal stromal tumors. J Clin Oncol 2002 15; 20: 3898-905

20. Shiu MH, Farr GH, Papachristou DN, Hadju SI. Myosarcomas of the stomach. Natural history, prognostic factors and management. Cancer 1982 (49): 177-87.

21. Bernet L, Bustamante M, Zúñiga A, Cano R. Characterization of GIST/GIPACT tumors by immunohistochemistry and exon 11 analysis of c-kit gene by PCR. Rev Esp Enferm Dig 2003; 95: 688-91.

22. Ng EH, Pllock RE, Munsell MF, Atkinson EN, Romsdahl MM. Prognostic factors influencing survival in gastrointestinal leiomyosarcomas. Implications for surgical management and staging. Ann Surg 1992: 69: 68-77.
23. Cabello Rodríguez M, Rueda Pérez JM, Somaza de Saint-Palais M, Fernández Lobato R. Leiomioblastoma. Tumor de histogénesis controvertida. Rev Esp Enferm Dig 1991; 80: 352-3.

24. DeMatteo RP, Lewis JJ, Leung D, Mudan SS, Woodruff JM, Brennan MF. Two hundred gastrointestinal stromal tumors: recurrence patterns and prognostic factors for survival. Ann Surg 2000 (231). 51-8.

25. Goss GA, Merriam P, Manola J, Singer S. Fletcher CD, Demetri GD. Clinical and pathological characteristics of gastrointestinal stromal tumors (GIST). Prog Proc Am Soc Clin Oncol 2000; (19): 599-abstract.

26. Solís-Herruzo JA, Solís-Muñoz P. GISTs: from the molecular knowledge to the racional treatment. Rev Esp Enferm Dig 2003 95: 677-82

27. Heinrich MC, Griffith DJ, Druker BJ, Wait CL, Oh KA, Zigler AJ. Inhibition of c-kit receptor tyrosine kinase activity by STI-571, a selective tyrosine kinase inhibitor. Blood 2000; 96: 925-32.

28. Heinrich MC, Corless CL, Duensing A, McGreevey L, Chen CL, Joseph $\mathrm{N}$, et al. PDGFRA activating mutations in gastrointestinal stromal tumors. Science 2003; 299 (5607): 708-10.

29. Tuveson DA, Willis NA, Jacks T, et al. STI-571 inactivation of the gastrointestinal stromal tumor c-KIT oncoprotein: biological and clinical implications. Oncogene 2001; 20: 5054-8.

30. Demetri GD, Von Mehren M, Blake CD, Van den Abbeele AD, et al Efficacy and safety of Imatinib Mesylate in advanced gastrointestinal stromal tumors. N Engl J Med 2002; 347: 472-80.

\title{
Tumores estromales gástricos: formas de presentación y opciones quirúrgicas
}

\author{
C. Pardo Martínez, J. Mayol Martínez, C. Hernández Pérez y J. Álvarez Fernández-Represa \\ Servicio de Cirugía General I. Hospital Clínico San Carlos. Madrid
}

\section{RESUMEN}

Los tumores estromales representan el 1-3\% de las neoplasias gástricas primitivas. Se pueden presentar a cualquier edad, bajo muy diversas formas clínicas, siendo raro que alcancen un tamaño superior a los $10 \mathrm{~cm}$. Estos raros tumores se encuadran en la actualidad dentro de los denominados tumores del estroma gastrointestinal (GIST's), cuya clasificación continúa siendo controvertida. La cirugía es el tratamiento de elección y su extensión viene determinada por el tamaño del tumor, la afectación de órganos vecinos y la presencia o no de metástasis. En casos seleccionados, la cirugía mínimamente invasiva puede llevarse a cabo con excelentes resultados.

Presentamos cuatro casos que resumen las distintas formas de presentación clínica de estos tumores así como su manejo terapéutico.

Palabras clave: Leiomioblastoma. Tumores estromales. GIST. Tratamiento. Cirugía.

\section{INTRODUCCIÓN}

Los tumores del estroma gastrointestinal (GIST) son extremadamente raros, representando sólo el 1-3\% de las neoplasias gástricas primitivas (1). Aparecen en cualquier grupo de edad, con una incidencia máxima en la sexta década de la vida. Su localización más frecuente es la parte media del estómago, seguida por el antro. Cerca del $20 \%$ de estos tumores se sitúan cerca del píloro, pero es raro que produzcan obstrucción (2). Sus dimensiones varían desde unos milímetros a decenas de centímetros, alcanzando sólo el $20 \%$ de ellos un tamaño superior a los $10 \mathrm{~cm}$. Entre el 30 y el $50 \%$ de los enfermos están asintomáticos en el momento del diagnóstico $(3,4)$, especialmente cuando la neoplasia es de pequeño tamaño. La dificultad para diagnosticar estos tumores con métodos no invasivos (5) ha sido en gran medida su- 
perada desde la aplicación de la TAC y la ecografía, que constituyen, junto con la endoscopia con biopsias múltiples (6) de la lesión sospechosa para su posterior estudio inmunohistoquímico, las pruebas diagnósticas más útiles y de empleo obligatorio en el diagnóstico y seguimiento de estos tumores.

La cirugía es su tratamiento de elección, aunque las opciones pueden variar desde las mínimamente invasivas hasta las grandes resecciones con el objeto de conseguir márgenes libres de enfermedad residual.

Presentamos cuatro casos clínicos que resumen el manejo diagnóstico y terapéutico actual de estos tumores.

\section{CASOS CLÍNICOS}

Caso 1. Varón de 76 años, asintomático. En una ecografía de rutina se objetiva una masa extrínseca que protuye en la pared del estómago. Se realiza una endoscopia (Fig. 1) que muestra una tumoración de unos $3 \mathrm{~cm}$ dependiente de la pared gástrica. Se decide tratamiento quirúrgico con resección gástrica en cuña por laparoscopia, bajo control endoscópico. El informe histológico describe una proliferación neoplásica de estirpe mesenquimal con escasas mitosis (menos de 1/50 CGA). Con técnicas de inmunohistoquímica, la lesión es negativa para la citoqueratina, la S-100, la desmina y la actina y positivas para la vimentina. El paciente está libre de enfermedad a los 2 años y medio de la intervención.

Caso 2. Mujer de 72 años, intervenida de forma programada tras hallar en una gastroscopia una tumoración en curvadura mayor gástrica, submucosa, ulcerada, de unos $4 \mathrm{~cm}$, compatible con tumor del estroma gastrointestinal maligno de tipo indeterminado. Se realizó una resección en cuña. A los 2 años es reintervenida por recidiva regional extragástrica detectada en la TAC de control, con gastroscopia normal. Se realiza antrectomía por afectación tumoral extraluminal junto con extirpación en bloque del epiplon mayor y bazo por infiltración tumoral. Hígado libre de metástasis. El estudio inmunohistológico describe implantes peritoneales, epiploicos, gástricos y metástasis linfoganglionares de tumor del estroma gastrointestinal maligno (15 mitosis por campo de gran aumento), positivo para CD34 y C-KIT (CD117) y negativo para marcadores musculoespecíficos (actina y desmina) y para la S-100, la sinaptofisina y la Leu-7. La enferma está libre de enfermedad a los 9 meses de la reintervención.

Caso 3. Varón de 71 años que acude a urgencias por un cuadro de 2 meses de evolución consistente en astenia, pérdida de peso y dolor abdominal intermitente. Se realiza una TAC, la cual identifica la presencia de una masa abdominal de unos 25x25x13 cm que ocupa la región epigástrica y mesogástrica, con áreas de necrosis en su interior y que comprime al estómago, sin poder definir su origen. El hígado está libre de lesiones. Se planifica el tratamiento quirúrgico, identificando una gran tumoración gástrica que obliga a realizar una gastrectomía subtotal junto con la resección del colon transverso que se encuentra infiltrado. El informe histológico describe una proliferación neoplásica de estirpe mesenquimal con 31 mitosis por 50 campos de gran aumen- to, cuyas células son negativas para la citoqueratina, la actina, la desmina y la S-100 y positivas para la vimentina. No existe afectación ganglionar. El paciente acude a urgencias a los 8 meses de la intervención por anorexia extrema y masa dolorosa en hemiabdomen superior. En la TAC (Fig. 2) se detecta recidiva local con metástasis ganglionares, retroperitoneales, mesentéricas, hepáticas y pulmonares. El paciente falleció un mes después.

Caso 4. Mujer de 60 años, hipertensa, que acude a urgencias por dolor abdominal de inicio brusco acompañado de vómitos y cuadro sincopal. Destaca tensión arterial 60/40, hemoglobina de $8,1 \mathrm{~g} / \mathrm{dl}$ y hematocrito de $24,7 \%$. La TAC (Fig. 3) confirmó la existencia de una masa mixta (sólida y líquida) de unos $20 \mathrm{~cm}$, dependiente de la cara posterior del estómago, ya detectada previamente en la ecografía abdominal inicial. La paciente fue intervenida de forma urgente, identificándose un abundante hemoperitoneo y una gran tumoración gástrica necrosada y sangrante que obligó a realizar una gastrectomía subtotal con resección de íleon terminal, colon ascendente y mitad proximal del colon transverso por afectación tumoral por vecindad. El estudio histopatológico de la tumoración la describe como una neoplasia constituida por células dispuestas en fascículos, con escasas mitosis (menos de 1/50 campo de gran aumento) y muy bajo índice proliferativo (Ki67). Las técnicas de inmuno- histoquímica demuestran negatividad para la actina, la desmina, la S-100 y la queratina y positividad para el CD-34 y la vimentina. La paciente está libre de enfermedad a los 18 meses de la intervención.

\section{DISCUSIÓN}

Los tumores del estroma gastrointestinal (GIST) constituyen la categoría más amplia de neoplasias no epiteliales primarias del estómago e intestino delgado (7-9). El término GIST, empleado por primera vez en 1983 por Mazur y Clark (10), engloba un grupo heterogéneo de neoplasias no epiteliales que muestran una gran variedad de diferenciaciones, lo que ha provocado un grado considerable de confusión en cuanto a su interpretación. En la década de los 80, diversos estudios inmunohistoquímicos mostraron que estos tumores pueden tener grados de diferenciación (11-14) muy variable, tanto hacia células del músculo liso (demostrable por la expresión de actina, miosina y desmina), como hacia elementos nerviosos o ganglionares autonómicos, así como presentar una doble diferenciación, neuronal y muscular, o permanecer indiferenciados.

En 1998, Kindblom (15) planteó por primera vez la teoría de que estos tumores derivasen de la misma estirpe celular que la célula intersticial de Cajal, basándose en la positividad en ambos tipos celulares de un mismo marcador, el CD117 (c-KIT).

Existen múltiples clasificaciones con valor pronóstico de estos tumores, siendo una de las más aceptadas la que incluye el tamaño tumoral, afectación ganglionar, metástasis a distancia y estudio inmunopatológico, revisada en 1993 por el UICC (16). Los factores pronósticos aceptados en el actualidad incluyen: localización del tumor a lo largo del trac- 
to gastrointestinal (10), tamaño tumoral superior a $5 \mathrm{~cm}$ (17), inmunohistoquímica del tumor (atipias nucleares, presencia de necrosis, invasión vascular, número de mitosis $>5 \times 50$ campos de gran aumento, mutación en el protooncogén c-KIT) $(18,19)$, así como la participación de órganos vecinos y la presencia de metástasis (20). Aunque diversos autores han encontrado una asociación entre las mutaciones del exón 11 del gen c-Kit y el comportamiento más agresivo de estos tumores, recientemente, Bernet y cols. (21) no fueron capaces de confirmar esta relación. También es considerado por algunos autores como factor de mal pronóstico la rotura tumoral durante su extirpación (22) así como la resección quirúrgica incompleta.

La localización más frecuente de las metástasis de estos tumores, como queda de manifiesto en el caso número 2, es el hígado, peritoneo y pulmón, pudiendo aparecer incluso hasta 30 años después de extirpar el tumor primario. La exéresis de las metástasis es aconsejable, dado que la neoplasia evolucionará, en general, más lentamente, incrementando la supervivencia del enfermo, que, en términos generales, no supera el $50 \%$ a los 5 años.

La cirugía es el tratamiento de elección de estos tumores, no existiendo diferencias significativas en términos de supervivencia a los 5 y 10 años (23) entre aquellos pacientes tratados con una amplia resección y los tratados con una mínima resección de la neoplasia, siempre que esta no supere los $5 \mathrm{~cm}$ de diámetro y no existan metástasis hepáticas en el momento del diagnóstico. Por esta razón, en pacientes seleccionados, como el presentado en el caso 1, la cirugía mínimamente invasiva tiene un importante papel con excelentes resultados.

Los tumores del estroma gastrointestinal no responden a la quimioterapia y radioterapia convencional $(24,25)$. Para aquellos enfermos en quienes la cirugía no ha pudo ser curativa, como tratamiento paliativo, se acaba de autorizar el uso del Imatinib Mesylato (Gleevec ${ }^{\circledR}$ ), empleado hasta ahora en el tratamiento de leucemia mieloide crónica con cromosoma Philadelphia (+). Su efecto terapéutico se basa en que la exposición de las células tumorales positivas para el receptor c-KIT a este fármaco, conduce al bloqueo de su actividad tirosin-quinasa, considerada como crítica en la patogenia de estos tumores. Este bloqueo detiene su proliferación (26) e induce la apoptosis (27).

Recientes estudios (28) han demostrado que existe un subgrupo de casos dentro de los GIST que carecen de la mutación c-KIT pero que presentan una mutación en el PDGFRA (receptor derivado del factor de crecimiento alfa derivado de las plaquetas, perteneciente a la familia de la tirosin-quinasa). Esta mutación es identificable mediante pruebas de inmunoprecipitación con antisueros policlonales dirigidos contra péptidos de secuencias específicas de la familia del receptor tirosin-quinasa. Ambas mutaciones, cKIT y PDGFRA, parecen ser alternativas y mutuamente excluyentes en los mecanismos oncogénicos de estos tumores. Por tanto, la ausencia de expresión de la mutación KIT no invalida el diagnóstico, debiendo ser considerado como un indicador de una posible mutación en el PDGFRA. Por este motivo, la ausencia de expresión del c-KIT no implica que un paciente con dicha neoplasia no pueda ser tratado con Gleevec $^{\circledR}(28)$. Este fármaco ha demostrado, tanto en modelos preclínicos (29) como en estudios clínicos (30), su efectividad en pacientes con GIST irresecables o metastáticos, constituyendo una esperanza terapéutica para dichos enfermos. 